\title{
Low-energy electron collisions with water: elastic and rotationally inelastic scattering
}

\author{
A. Faure, J. D. Gorfinkiel and Jonathan Tennyson \\ Department of Physics and Astronomy, University College London, Gower Street, \\ London WC1E 6BT, UK
}

\begin{abstract}
.
Differential, integral and momentum transfer cross sections for the vibrationally elastic and rotationally inelastic scattering of electrons from water at low collision energy $(\mathrm{E}<7 \mathrm{eV})$ are reported. The $R$-matrix method is used to compute the bodyfixed $T$-matrices while the scattering calculations are performed within the fixed-nuclei approximation corrected with the standard Born-closure formula. Our calculations are compared with the very recent experimental results of Cho et al (Cho H, Lee H S and Park Y S 2003 Radiat. Phys. Chem. 68 115). The differential and momentum transfer cross sections are in good agreement with the experimental results. The relative contribution of the rotationally inelastic processes is investigated in some details. In particular, the importance of the pure elastic process at very low energy is emphasized.
\end{abstract}

PACS numbers:

\section{Introduction}

Water is an extremely important molecule: it is ubiquitous in the universe and fundamental to life. Collisions of electrons with water molecules play thus an important role in a variety of research fields such as astrophysics and atmospheric physics, radiation biology and plasma physics. In the last two decades, these collisions have been studied extensively, both experimentally and theoretically (for a review, see Mason and Itikawa (2004)). In particular, differential cross sections (DCS) for the vibrationally elastic scattering have been computed and measured by several authors for collision energies below $50 \mathrm{eV}$. Elastic DCS of water indeed provide crucial parameters for modelling the radiation effects on biological matter (e.g. Champion 2003). The recent experimental paper by Cho et al (2003) gives absolute DCS at four incident energies between 4 and $50 \mathrm{eV}$ and over scattering angles of $10-180^{\circ}$ measured with an original device to extend the measurements to backward angles. A detailed comparison of this data with previous measurements and calculations shows general good agreement among the experimental data up to $100^{\circ}$. At higher angles, however, the available data show very different behaviours. In particular, the results of Cho et al (2003) are consistently lower than previous experimental DCS in the backward direction. The elastic integral cross section (ICS) and the momentum transfer cross section (MTCS) were also derived by 
Cho et al (2003) by extrapolating the DCS at the forward scattering angles that are difficult to measure experimentally. As discussed by Okamoto et al (1993), such an extrapolation procedure introduces rather large uncertainties because the large dipole moment of water leads to heavily forward peaked cross sections. Reliable theoretical DCS for the forward scattering are therefore highly desirable, as we shall further discuss below.

In this paper, we report differential, integral and momentum transfer cross sections for vibrationally elastic electron scattering from water at collision energies below $7 \mathrm{eV}$. The water molecule is described by an ab initio multicentred wavefunction using the $R$-matrix method while the scattering calculations are performed within the fixednuclei (FN) approximation. A comparison with the most recent experimental results is presented. The theoretical and computational treatment is briefly introduced in the next section. Results are discussed in section 3. Conclusions are summarized in section 4.

\section{Theory}

All calculations were performed using the electron- $\mathrm{H}_{2} \mathrm{O}$ wavefunctions developed by Gorfinkiel et al (2002) who calculated cross sections for different molecular geometries (see paper for full details). In the present paper, the $R$-matrix calculations were performed at the equilibrium geometry of $\mathrm{H}_{2} \mathrm{O}\left(r_{\mathrm{OH}}=1.81 a_{0}\right.$ and $\left.\widehat{H O H}=104.5^{\circ}\right)$ using an $R$-matrix sphere of radius $10 a_{0}$. The total wavefunction takes the form:

$$
\Psi_{k}=\sum_{i j} a_{i j k} \phi_{i}\left(x_{1}, \ldots, x_{N}\right) u_{i j}\left(x_{N+1}\right)+\sum_{i} b_{i k} \chi_{i}\left(x_{1}, \ldots, x_{N+1}\right),
$$

where $\phi_{i}$ are target wavefunctions, $u_{i j}(x)$ are continuum orbitals and $\chi_{i}$ are two-centre quadrically integrable $\left(L^{2}\right)$ functions constructed from the target occupied and virtual molecular orbitals. These last functions are important both for relaxing the enforced orthogonality between the target and continuum orbitals, and for representing shortrange polarization effects not included in the truncated partial wave expansion. The present scattering model includes the seven lowest electronic states of water generated using a CASCI (complete active space configuration interaction) procedure and averaged natural orbitals. This model gives a dipole moment of $1.864 \mathrm{D}$, which can be compared to the experimental value of 1.854 D (Suresh and Naik 2000). The continuum orbitals were represented using the Gaussian-type orbitals basis set developed by Faure et al (2002) which include all angular momentum up to $l=4$ and is optimized to span energies below $68 \mathrm{eV}$.

Cross sections were calculated following the procedure implemented in the program POLYDCS (Sanna and Gianturco 1998). The general theory of the scattering of an electron from a polyatomic molecule in the fixed-nuclei $(\mathrm{FN})$ approximation has been presented many times before, see for example the review by Gianturco and Jain (1986). In this approach, the cross section is expressed as a partial-wave expansion within the adiabaticnuclei-rotation (ANR) approximation which assumes that the initial and final target states are degenerate (Lane 1980). For low partial-waves (here $l \leq 4$ ), the cross section is 
computed from the FN $T$-matrices obtained via the $R$-matrix calculations. In the case of a polar molecule, the partial-wave expansion does not converge in the FN approximation, owing to the very long-range nature of the electron-dipole interaction. To circumvent this problem, the standard procedure is to use the dipolar Born approximation to obtain the cross section for the high partial-waves not included in the FN T-matrices. The final cross section is then calculated as the sum of two contributions and can be regarded as a short-range correction to the Born approximation. The DCS is thus given by the familiar expression (e.g. Gianturco et al 1998):

$$
\frac{d \sigma}{d \Omega}={\frac{d \sigma^{B}}{d \Omega}}^{B}+\sum_{L}\left(A_{L}-A_{L}^{B}\right) P_{L}(\cos \theta),
$$

where $P_{L}(\cos \theta)$ is the Legendre function and $A_{L}$ are coefficients which depend explicitly on products of $T$-matrix elements and on algebraic factors. The superscript $B$ denotes that the relevant quantity is calculated within the dipolar Born approximation. The ICS and MTCS are calculated similarly by

$$
\sigma=\sigma^{B}+4 \pi\left(A_{0}-A_{0}^{B}\right) .
$$

and

$$
\sigma_{m}=\sigma_{m}^{B}+4 \pi\left(A_{0}-A_{0}^{B}\right)-\frac{4}{3 \pi}\left(A_{1}-A_{1}^{B}\right) .
$$

It should be noted that the present cross sections correspond to the vibrationally elastic ones, which are summed over all final rotational states. As shown by Okamoto et al (1993), $\frac{d \sigma}{d \Omega}$ does not depend on the initial rotational state of the target molecule unless the scattering angle is very close to $0^{\circ}$. This means that theoretical DCS can be computed for the target in its ground rotational state, whatever the (rotational) temperature at which the experiment was performed. The averaging over a particular rotational state distribution would appear necessary at energies close to rotational thresholds, where the ANR approximation becomes invalid (Morrison 1988). In the following, cross sections have therefore been calculated for the water molecule in its ground rotational state $(j \tau)=(00)$.

\section{Results}

A comparison between our calculations and the experimental results of Cho et al (2003) is presented in figure 1, where the elastic (rotationally summed) DCS is plotted for a collision energy of $4 \mathrm{eV}$. The first electronic excitation threshold of $\mathrm{H}_{2} \mathrm{O}$ at equilibrium geometry is around $7 \mathrm{eV}$. Three Feshbach resonances, the lowest lying just below the first threshold, were found for $\mathrm{E}<16 \mathrm{eV}$ (Gorfinkiel et al 2002); their positions are strongly dependent on molecular geometry. Hence, above $7 \mathrm{eV}$, electronic excitation and geometry effects will start to have some effect. For this reason, we made no attempt to investigate the other energies $(15,40$ and $50 \mathrm{eV})$ reported by Cho et al (2003). It can be noticed that our calculation reproduces the experimental data very well, especially for backward angles $\left(130-180^{\circ}\right)$, where previous experimental data were consistently 
higher than the present results. Comparisons for intermediate angles $\left(10-120^{\circ}\right)$ at $6 \mathrm{eV}$ with the experimental results of Johnstone and Newell (1991) and the theoretical results of Gianturco et al (1998) give good agreement (Faure et al 2003).

Also shown in figure 1 are the partial state-to-state $\frac{d \sigma}{d \Omega}\left(00 \rightarrow j^{\prime} \tau^{\prime}\right)$ summed over the $\tau^{\prime}$ pseudo quantum number. The expected dominant contribution of the dipolar $(0 \rightarrow 1)$ rotational transition at forward angles is clearly shown. However, in contrast to the higher energy calculations by Gianturco et al (1998), the pure elastic $(0 \rightarrow 0)$ transition becomes dominant at backward angles. This result indicates the crucial role of the shortrange interactions, as described by the low-partial waves, in the backward scattering region. In the minimum region $\left(120-140^{\circ}\right)$, we notice that the $(0 \rightarrow 2)$ transition is also important.

Figure 2 presents the vibrationally elastic ICS, as well as partial state-to-state ICS with $\Delta j=0-5$ for collision energies in the range $0.1-7 \mathrm{eV}$. We notice, again, that the $\Delta j=1$ rotational excitation is the most efficient process in the whole range of collision energies. However, it is interesting to note that below about $3 \mathrm{eV}$, the relative contribution of the pure elastic $\Delta j=0$ process to the total ICS increases sharply with decreasing energy. Thümmel et al (1992) showed in their calculations for vibrationally elastic scattering of HF including non-adiabatic rotational effects that the pure elastic ICS will become larger than the $\Delta j=1$ ICS at energies close to rotational thresholds. As noted above, the ANR approximation fails near rotational thresholds where a full rotational close-coupling calculation would indeed be necessary. We have consequently restricted the present calculations to energies larger than $100 \mathrm{meV}$. In any case, it is worth noting that our results are in disagreement with the usual assumption that the pure elastic scattering process is negligible at very low energy (e.g Field et al 2000).

At energies above $3 \mathrm{eV}$, the $\sigma(0 \rightarrow 0)$ cross section is found to display a pronounced rise which is probably caused by the Feshbach resonance of ${ }^{2} \mathrm{~B}_{1}$ symmetry that lies just below the first excited electronic state (Gorfinkiel et al 2002). As mentioned earlier, the position of this resonance depends strongly on the molecular geometry. Therefore, inclusion of vibrational motion is likely to have a strong effect on the cross section in the resonace region. ICS for higher rotational transitions $(\Delta j=2,3,4)$ are also affected, but to a lesser extent, by this resonance (see figure 2 ).

The ICS determined experimentally by Cho et al (2003) at $4 \mathrm{eV}$ is lower than ours by a factor of 3 . Since the experimental and calculated DCS are in good agreement over the range of experimental measurements, this difference seems to come from the extrapolation procedure used by Cho et al (2003) at forward angles, where the DCS increases very steeply owing to the dipole interaction. This problem, previously highlighted by Okamoto et al (1993) and Gianturco et al (1998), indicates that ICS deduced from experiment in this fashion are not reliable. Figure 3 presents the vibrationally elastic MTCS as a function of the collision energy. In contrast to the ICS, our results are in good agreement with the value determined by Cho et al (2003). This probably reflects the fact that the forward angle contribution of the DCS to the MTCS is small. Conversely, the difference with the results of Johnstone and Newell (1991) 
emphasises the importance of the backward direction. Our calculated MTCS is also found to possess a minimum value of $6.46 \AA^{2}$ at about $4 \mathrm{eV}$, again probably caused by the ${ }^{2} \mathrm{~B}_{1}$ resonance. It is interesting to note that such a feature in the MTCS (minimum value of $6 \AA^{2}$ at about $3 \mathrm{eV}$ ) was reported by Hayashi (1989) on the basis of various theoretical and experimental data in the energy range $1-50 \mathrm{eV}$.

\section{Conclusions}

Our calculated cross sections are en excellent agreement with the latest differential measurements. The results show that extrapolation of experimental cross sections to small angles still causes problems. The low energy importance of the rotationally elastic process, which is often neglected because it is equal to zero in the pure dipolar approximation, is also shown. Neither this process, nor the $(0 \rightarrow 2)$ transition can be neglected at low energies.

\section{Acknowledgments}

We are grateful to Prof. Y. Itikawa who encouraged us to carry out these calculations.

\section{References}

Champion C 2003 Phys. Med. Biol. 482147

Cho H, Lee H S and Park Y S 2003 Radiat. Phys. Chem. 68115

Faure A, Gorfinkiel J D, Morgan L A and Tennyson J 2002 Comput. Phys. Commun. 144224

Faure A, Gorfinkiel J D and Tennyson J Mon. Not. R. Astron. Soc., in press

Field D, Jones N C, Mason N J, Lunt S L and Ziesel J P 2000 J. Phys. B.: At. Mol. Opt. Phys. 33 1039

Gianturco F A and Jain A 1986 Phys. Rep. 143347

Gianturco F A, Meloni S, Paioletti P, Lucchese R R and Sanna N 1998 J. Chem. Phys. 1084002

Gorfinkiel J D, Morgan L A and Tennyson J 2002 J. Phys. B.: At. Mol. Opt. Phys. 35543

Hayashi M 1989 Atomic and Molecular Data for Radiotherapy Proc. IAES Advisory Group Meeting, Vienna Report No IAEA-TECDOC-506, p. 193

Johnstone W M and Newell W R 1991 J. Phys. B.: At. Mol. Opt. Phys. 243633

Lane N F 1980 Rev. Mod. Phys. 5229

Mason N J, Itikawa Y, in preparation

Morrison M A 1988 Adv. At. Mol. Phys. 2451

Okamoto Y, Onda K and Itikawa Y 1993 J. Phys. B.: At. Mol. Opt. Phys. 26745

Sanna N, Gianturco 1998 Comput. Phys. Commun. 114142

Suresh S J and Naik V M 2000, J. Chem. Phys. 1139727

Thümmel H T, Nesbet R K and Peyerimhoff S D 1992 J. Phys. B.: At. Mol. Opt. Phys. 254553 


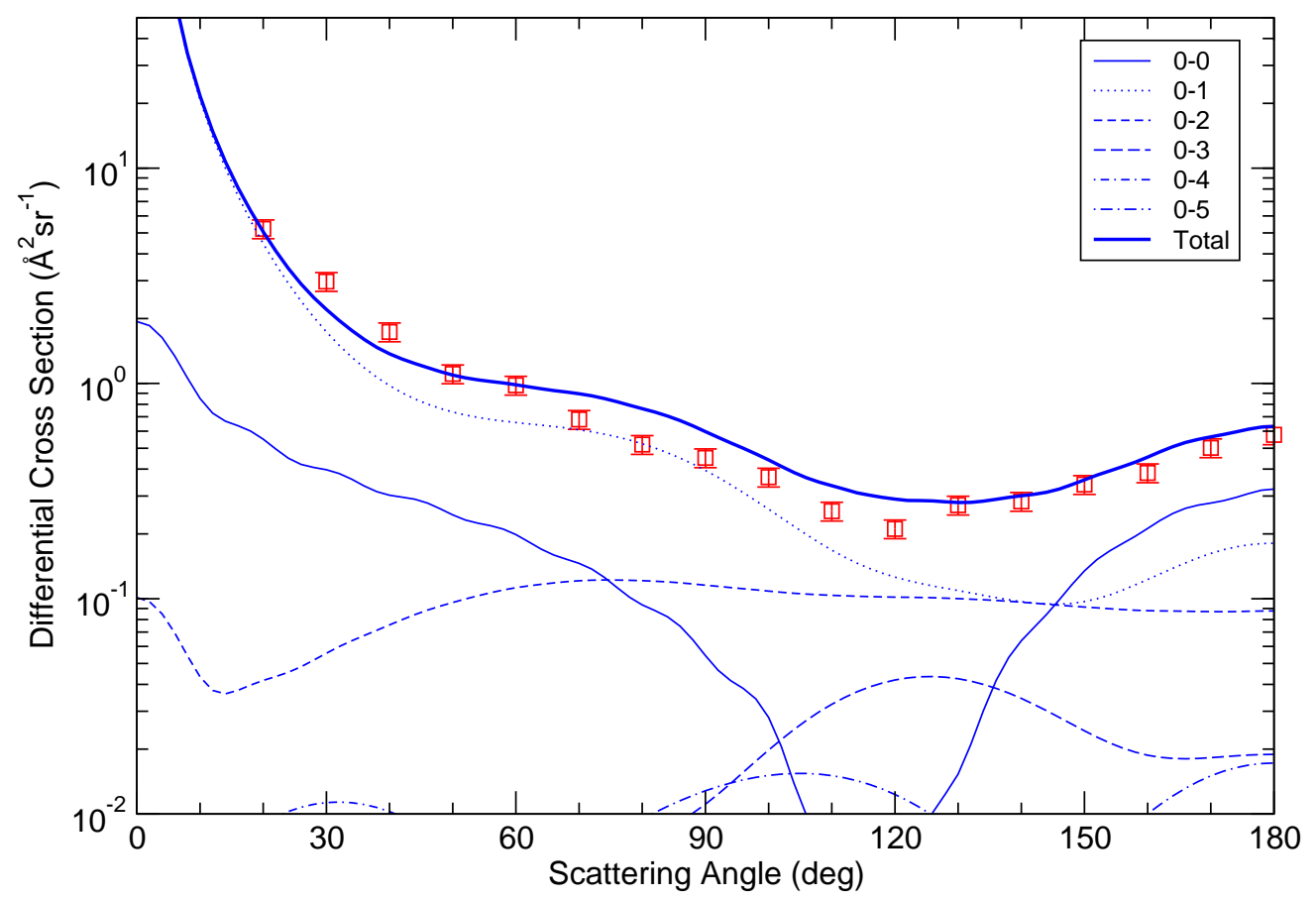

Figure 1. Elastic (rotationally summed) DCS of water at $4 \mathrm{eV}$. The squares are the experimental points from Cho et al (2003). The present calculation is given by the thick solid line. Other lines denote partial state-to-state DCS.

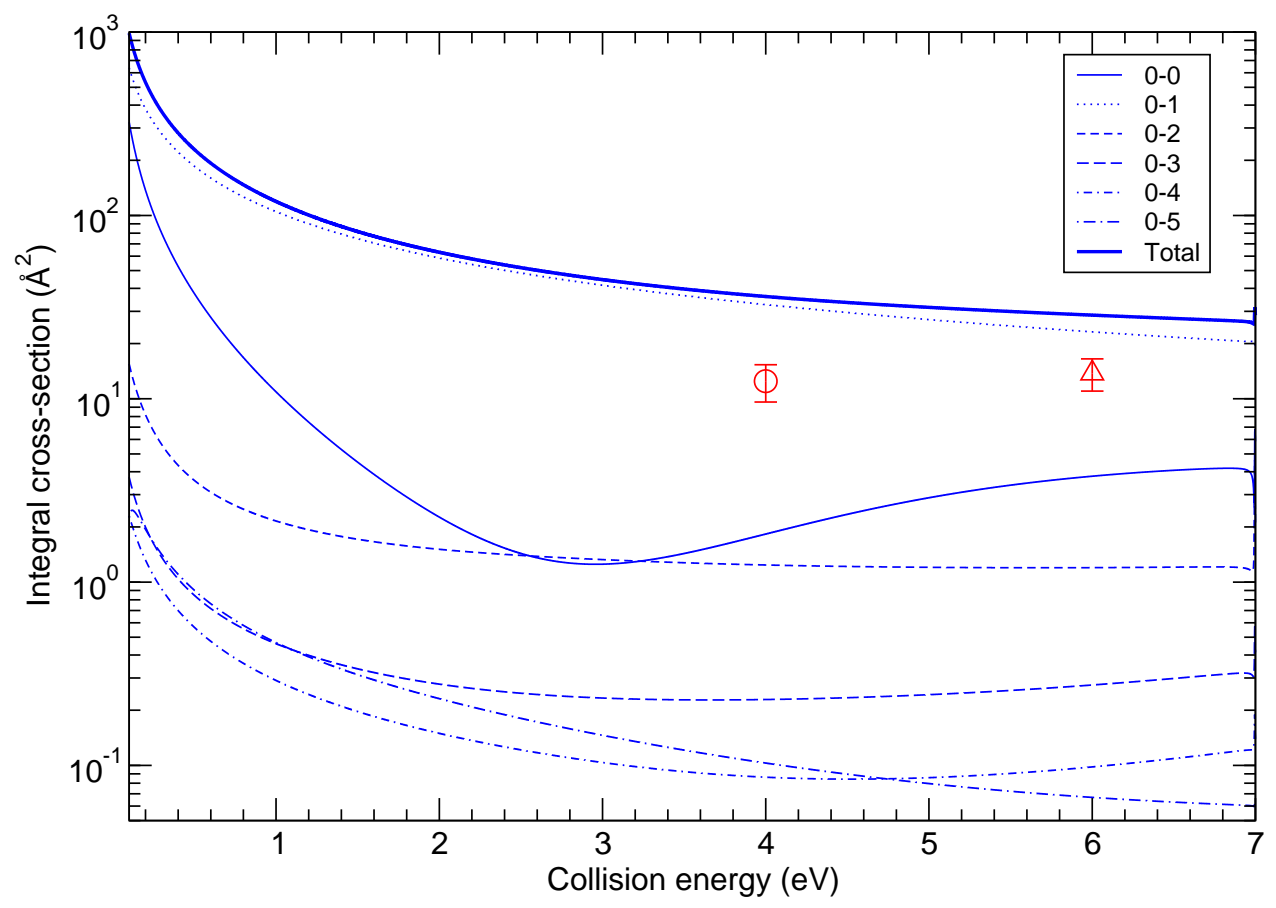

Figure 2. Elastic (rotationally summed) ICS of water as a function of the collision energy. The experimental determination of Cho et al (2003) (open circle) and Johnstone and Newell (1991) (open triangle) are plotted for comparison. Partial stateto-state ICS are also shown. 


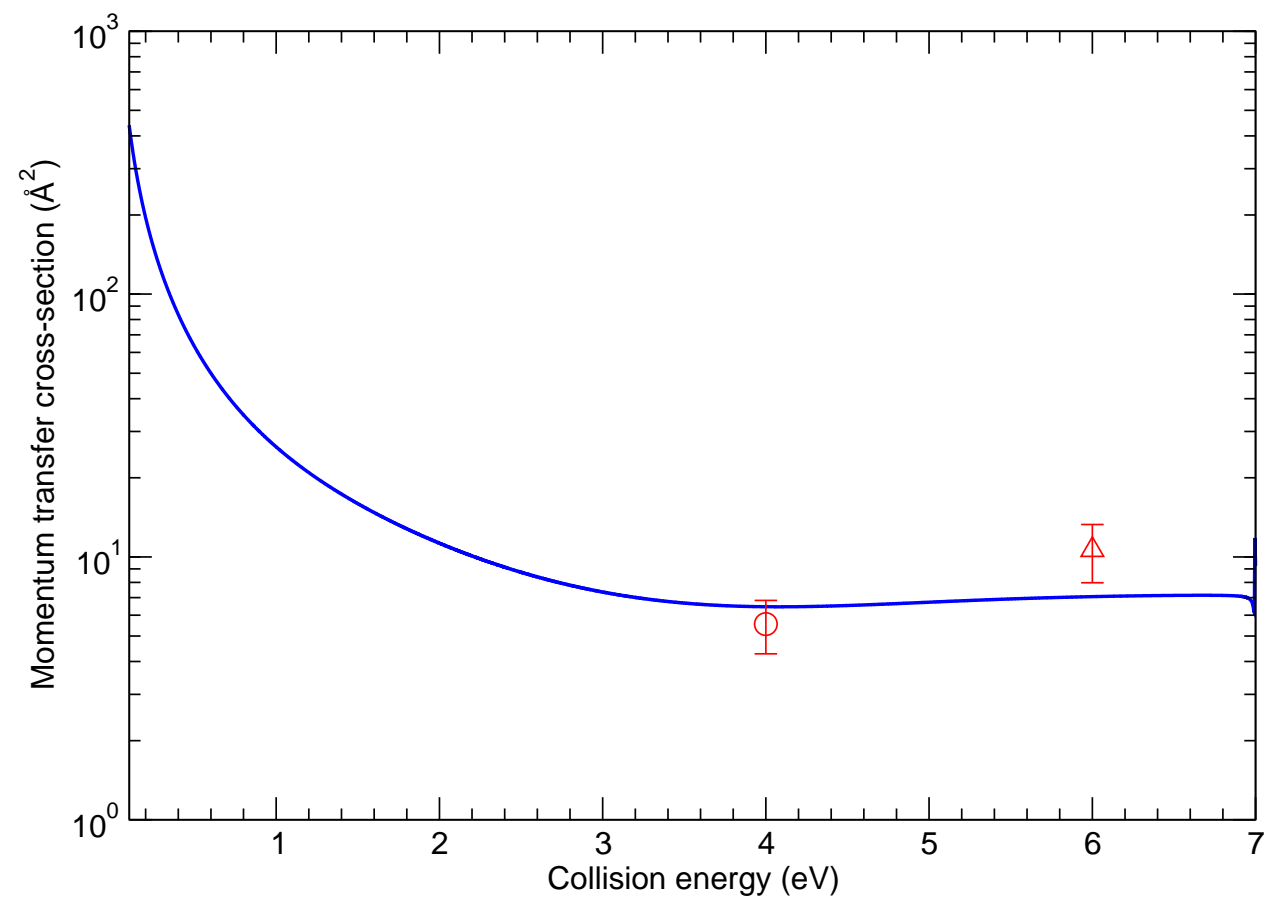

Figure 3. Elastic (rotationally summed) MTCS of water as a function of the collision energy. The experimental determination of Cho et al (2003) (open circle) and Johnstone and Newell (1991) (open triangle) are plotted for comparison. 